\title{
SUPERVISANDO LA SEGURIDAD VIAL EN EL PERÚ
}

\author{
Freddy Sagástegui ${ }^{1}$
}

\begin{abstract}
RESUMEN
En este artículo se describe algunos problemas en seguridad vial que la Defensoría del Pueblo del Perú, en su trabajo como ente supervisor de la administración pública ha detectado, entre esos problemas se mencionan a: la escasa y mala elaboración de estadísticas de accidentes de tránsito; la inconsistencia del Consejo Nacional de Seguridad Vial y la irresponsabilidad de los gobiernos regionales y locales por su falta de aplicación del Plan Nacional de Seguridad Vial.
\end{abstract}

Ante ello, la Defensoría del Pueblo sugiere que el Estado desarrolle un sistema estadístico riguroso, que fortalezca al Consejo Nacional de Seguridad Vial y que los diferentes sectores del Estado desarrollen el mencionado Plan Nacional.

Palabras clave: Accidentes de tránsito; Sistemas de información; Gobierno; Salud pública; Perú (fuente: DeCS BIREME).

\section{SUPERVISING ROAD SAFETY IN PERU}

\begin{abstract}
In this article some problems in road safety are described, which have been detected by the Ombudsman of Peru, as part of its role of public administration supervisor, amongst these problems we mention: lack of and inadequate elaboration of the statistics on road traffic accidents, the inconsistency of the National Council for Road Security and the lack of responsibility of the local and regional governments reflected by the lack of application of the National Plan of Road Safety. Facing this, the Ombudsman suggests that the State develops a rigorous statistical system, strengthens the National Council of Road Safety and that the different sectors of the government develop the mentioned National Plan.
\end{abstract}

Key words: Accidents, traffic; Information systems; Government; Public health; Peru (source: MeSH NLM).

\section{INTRODUCCIÓN}

Anualmente en el mundo, fallecen en promedio, 1,2 millones de personas, mientras que otras 50 millones quedan heridas producto de los accidentes de tránsito, por ello, los accidentes se han convertido en un mal que padecen todas las sociedades. Así, tenemos que en el año 1990, los accidentes de tránsito se encontraban en la novena posición en el rango de las diez causas principales de la carga mundial de morbilidad; sin embargo, se estima que para el año 2020 los accidentes de tránsito ocuparán el tercer lugar ${ }^{(1)}$.

Resulta difícil medir el nivel de daño que producen los accidentes en las sociedades y, sobre todo, en las familias, por cuanto todas las personas que mueren, se lesionan o quedan discapacitadas por un choque tienen personas allegadas, como familiares y amigos, que resultan profundamente afectadas ${ }^{(1,2)}$.

Existe un grave problema que afecta a los familiares de quienes fallecen en los accidentes, es así que en el año
2002 se estimó que más de la mitad de los fallecidos a nivel mundial fueron personas entre 15 a 44 años, es decir en la época económicamente más productiva de su vida, hecho que perjudica gravemente a la familia pues sufren pérdida de ingresos.

En este sentido, la Defensoría del Pueblo como organismo constitucional autónomo y que tiene como mandato constitucional la defensa de los derechos constitucionales y fundamentales de la persona y la comunidad, supervisar el cumplimiento de los deberes de la administración estatal y la adecuada prestación de los servicios públicos a la ciudadanía, desarrolla un trabajo de supervisión a las autoridades encargadas de la seguridad vial en el país con la finalidad de realizar diagnósticos y proponer sugerencias a las autoridades, con la finalidad de contribuir a generar una cultura de seguridad vial en el Perú.

En este artículo se analiza dos puntos, el primero está vinculado con la defectuosa estadística de los accidentes de tránsito la que no permite alertar al Estado sobre las

${ }_{1}^{1}$ Abogado, Adjuntía para el Medio Ambiente, Servicios Públicos y Pueblos Indígenas, Defensoría del Pueblo. Lima, Perú.

Recibido: 14-03-10 Aprobado: 19-05-10 
principales variables de los accidentes; el segundo está asociado con la inconsistencia del Consejo Nacional de Seguridad Vial como órgano encargado de la seguridad vial en el país y la necesidad de que los gobiernos regionales y locales se comprometan con el Plan Nacional de Seguridad Vial.

\section{MEJORAR LA ESTADÍSTICA OFICIAL DE LOS ACCIDENTES DE TRÁNSITO}

Al revisar la estadística nacional de accidentes de tránsito de los últimos diez años en el Perú, vamos a encontrarnos con las cifras que se muestran en la Tabla 1.

En los datos mostrados no se observa que haya tendencia decreciente o de estancamiento de los accidentes de tránsito en el país, lo mismo sucede en las cifras de fallecidos y heridos, es más, en los últimos diez años prácticamente se ha duplicado la cifra de heridos.

Sin embargo, la Defensoría del Pueblo ha encontrado varios problemas en estos datos, en primer lugar, no es una estadística rigurosa, dado que no se consigna a los heridos que luego del accidente fallecen; es decir, no se cumplen las recomendaciones de la Organización Mundial de la Salud (OMS) que plantea hacer un seguimiento a los afectados en los accidentes de tránsito durante los 30 días posteriores al accidente.

Este problema ya fue consignado en el Informe Defensorial N. ${ }^{\circ}$ 137: "El transporte urbano en Lima Metropolitana: un desafío en defensa de la vida", en el

Tabla 1. Cifras de accidentes de tránsito en el Perú 1998-2008.

\begin{tabular}{cccc}
\hline Año & $\begin{array}{c}\mathbf{N} .^{\mathbf{0}} \mathbf{d e} \\
\text { accidentes }\end{array}$ & $\begin{array}{c}\mathbf{N}^{\circ} \mathbf{d e} \\
\text { muertos }\end{array}$ & $\begin{array}{c}\mathbf{N} .^{\circ} \text { de } \\
\text { heridos }\end{array}$ \\
\hline 1998 & 81115 & 3321 & 26417 \\
\hline 1999 & 79605 & 3214 & 31578 \\
\hline 2000 & 76665 & 3118 & 29945 \\
\hline 2001 & 76545 & 3200 & 27747 \\
\hline 2002 & 74221 & 2929 & 29887 \\
\hline 2003 & 74612 & 2856 & 32670 \\
\hline 2004 & 74401 & 3103 & 34752 \\
\hline 2005 & 74870 & 3302 & 39559 \\
\hline 2006 & 77840 & 3481 & 46882 \\
\hline 2007 & 79972 & 3510 & 49057 \\
\hline 2008 & 85337 & 3489 & 50059 \\
\hline
\end{tabular}

Fuente: Ministerio de Transportes y Comunicaciones. cual se menciona que los datos estadísticos de la Policía Nacional presentan un conjunto de problemas como ${ }^{(3)}$ :

- Se diferencia a las víctimas como mayores o menores de edad, sin disgregar en otros niveles como: adulto mayor, niño, adolescente o estudiantes y escolares, entre otros.

- Los datos no hacen una clara distinción si los vehículos que participan en los accidentes son vehículos de transporte público o vehículos privados; tampoco, por ejemplo, distinguen a los vehículos que brindan el servicio de auto-colectivo pese a que es una modalidad de transporte urbana usada por un sector de la población.

- No existe datos sobre lugares en Lima donde con frecuencia se presentan accidentes de tránsito no fatales (accidentes que sólo producen heridos) dado que la VII Dirección Territorial de la PNP no desarrolla este punto.

- Existen temas que son desarrollados por la Policía de Tránsito en los accidentes fatales, pero no son desarrollados por la VII Dirección Territorial de la PNP en los accidentes no fatales, por ejemplo, el ítem "estado de ebriedad del peatón".

El contar con datos estadísticos detallados de accidentes de tránsito, nos permitirá conocer con certeza qué viene ocurriendo con la siniestralidad en el país; así, estos datos nos servirán de alerta sobre los principales problemas que se tienen que trabajar con miras a reducir los accidentes de tránsito. También nos debe ayudar a que el Estado adopte las medidas que se requieren para evitar el incremento de dichos accidentes en determinados sectores. A manera de referencia, España ha elaborado un plan estratégico de seguridad vial aplicado a las motocicletas, una primera impresión nos llevaría a pensar que el público al que va dirigido este plan son los jóvenes de edades que deben fluctuar entre 18-23 años y que son usuarios frecuentes de este tipo de vehículo; sin embargo, el público al que esta orientado son adultos de 35-40 años porque es éste sector de la población el que más participación tiene en los accidentes en este tipo de vehículo.

Adicionalmente, estos datos estadísticos deben ser complementados con estudios para poder entender qué sucede realmente en los indicadores de accidentes de tránsito, y que ello nos permita concluir con certeza, cuáles son las reales causas de estos accidentes; así, un error común que se comete cuando se revisa la estadística de los accidentes es pensar que los peatones somos muy irresponsables en la vía pública, pero si consideramos el estudio "La vulnerabilidad de los peatones en la vialidad del área Metropolitana de 
Lima y Callao" elaborado por el Consejo de Transporte de Lima y Callao podremos verificar que en varias de las zonas denominadas puntos negros (zonas frecuentes de accidentes de tránsito) las vías han sido diseñadas de tal forma que se favorece la circulación de los vehículos automotores en desmedro de los peatones; así, el Consejo de Transporte de Lima y Callao detectó los siguientes problemas ${ }^{(4)}$ :

- Insuficiente semaforización para peatones.

- Conflicto de flujos vehiculares con flujos peatonales en el momento del giro a la derecha y a la izquierda.

- Aceras de espacio reducido frente a la gran cantidad de peatones.

- Peatones usan la calzada ante la falta de paraderos.

- Inexistencia o mala ubicación de los puentes peatonales para los cruces de vías.

- Inexistencia o escaso mantenimiento de la señalización preventiva o regulatoria.

- En las vías no existe preferencia al peatón.

- Los vehículos no respetan los cruces peatonales.

Por ello, es importancia que el Estado implemente un sistema de información de accidentes de tránsito que permita no sólo ser riguroso, sino también completo en información y, por tanto, ayude al Estado en adoptar decisiones para reducir los accidentes de tránsito.

\section{FORTALECIMIENTO DEL CONSEJO NACIONAL DE SEGURIDAD VIAL E IMPLEMENTACIÓN DEL PLAN NACIONAL DE SEGURIDAD VIAL}

En el Informe Defensorial N. ${ }^{0} 108$ : "Pasajeros en riesgo: la seguridad en el transporte interprovincial" (5), se constató la inconsistencia del Consejo Nacional de Seguridad Vial, el cual es un ente multisectorial conformado por el Ministerio de Transportes y Comunicaciones, el Ministerio de Salud, el Ministerio del Interior, el Ministerio de Educación, el Ministerio de Trabajo y Promoción del Empleo, la Superintendencia Nacional de Administración Tributaria (SUNAT), el Instituto Nacional de Defensa de la Competencia y de la Propiedad Intelectual (INDECOPI), la Asamblea Nacional de Gobiernos Regionales y la Municipalidad Metropolitana de Lima.

Este Consejo Nacional tiene entre sus principales funciones: proponer planes, metas y objetivos en seguridad vial, formular políticas de prevención de accidentes de tránsito, impulsar y evaluar la realización de acciones para la seguridad vial, proponer normas legales para el mejoramiento de la seguridad vial, promover la investigación sobre accidentes de tránsito, coordinar el trabajo de las organizaciones que participan en la seguridad vial, impulsar la implementación de programas de educación vial, organizar actividades y campañas de seguridad vial, entre otros ${ }^{(6)}$.

El Consejo tiene una secretaría técnica que cumple funciones ejecutivas, pero hasta el año 2006 solamente contaba con cuatro funcionarios y un presupuesto de S/. 298000 para desarrollar sus funciones y sólo en el año 2010 ha aumentado el número de funcionarios a 16 trabajadores con un presupuesto de cerca de dos millones de soles, el objetivo es no sólo mantener estos indicadores, sino mejorarlos con miras a poder realizar un adecuado trabajo de seguridad vial a nivel nacional y con metas de mediano y largo plazo.

Esta situación dista mucho de instituciones similares en otros países, por ejemplo Comisión Nacional de Seguridad de Tránsito (CONASET) de Chile cuenta con más de 20 funcionarios y un presupuesto promedio de $1 \mathrm{mi}-$ llón de dólares. Estos medios le permite a la CONASET, brindar asesoría técnica a los municipios, realizar estudios como el Manual de Recomendaciones de Elementos de Vialidad Urbana, realizar análisis de seguridad de tránsito mediante la aplicación del Índice de Seguridad de Tránsito (INSETRA), el tratamiento de puntos negros como medidas correctivas de bajo costo, realizar campañas de sensibilización a la población (por ejemplo la campaña "Aún te espero") y elaborar cartillas informativas vinculadas con la seguridad y educación vial ${ }^{(7)}$.

En consecuencia, es imperativo que el Estado fortalezca al Consejo Nacional de Seguridad Vial, brindándole el personal necesario y los recursos económicos que le permitan desarrollar un trabajo de seguridad vial a largo plazo en el país. En este sentido, es un avance la emisión del Decreto Supremo N. ${ }^{\circ}$ 013-2007-MTC, publicado el 26.04.07, mediante el cual se aprobó el Plan Nacional de Seguridad Vial 2007-2011 ${ }^{(8)}$, que es el primer plan nacional con objetivos a mediano plazo y que tienen como meta reducir el $30 \%$ de los accidentes de tránsito en dicho periodo.

Entre los principales objetivos estratégicos que desarrolla el Plan Nacional para cumplir la meta antes referida tenemos:

1. Educación y comunicación, para lo cual es necesario implementar un programa de educación vial en el sistema educativo nacional.

2. Desarrollo e investigación de la seguridad vial. Para ello se debe implementar un sistema de recolección de datos de colisiones de tránsito. 
3. Restablecer el sistema de revisiones técnicas de los vehículos.

4. Implementación del programa para la detección y eliminación de puntos negros en las vías (zonas donde frecuentemente ocurren accidentes de tránsito).

5. Diseño e implementación del programa de comunicaciones en materia de seguridad vial.

6. Ejecución del estudio de mejoramiento del sistema de emergencia, rescate y calidad en la asistencia de heridos.

7. Ejecución del estudio que determine los costos sociales de los accidentes de tránsito en el país.

8. Implementación de programas de auditorias viales, con la finalidad de corregir los errores en el diseño de las vías y que pueden contribuir a los accidentes de tránsito.

9. Programa de fortalecimiento del accionar policial para el control y fiscalización de las normas de tránsito. Para ello se debe dotar de las herramientas tecnológicas a la Policía Nacional (alcoholímetro, medidor de velocidad, etc.) para un mejor cumplimiento de sus funciones.

10. Ordenamiento del sistema de transporte público de pasajeros, optimizando la adecuada prestación del servicio de transporte urbano, reduciéndose la sobreoferta de esta modalidad de transporte así como su informalidad.

11. Coordinación y liderazgo del Plan Nacional de Seguridad Vial, lo cual implica incorporar el Plan Nacional a los planes operativos de los ministerios del Interior, Salud, Educación y Transportes, así como en los gobiernos regionales y locales.

12. Consolidación de la imagen del Consejo Nacional de Seguridad Vial en el país.

Sin embargo, difícilmente este plan tendrá éxito si no logramos empoderar al Consejo Nacional de Seguridad Vial en el país. Además, para el éxito del Plan Nacional se requiere el compromiso de los gobiernos regionales y locales, el desarrollo de líneas de trabajo importantes como: implementar programas para la detección y eliminación de los puntos negros, realizar auditorias viales en vías regionales y locales, ordenamiento de los sistemas de transporte regional y urbano, eliminando la sobreoferta y combatiendo la informalidad en estas modalidades de transporte, entre otros.

No obstante, la realidad nos demuestra que estos niveles de gobierno no se han comprometido con el plan nacional, prueba de ello es el caso de la Municipalidad Metropolitana de Lima que no ha realizado estrategia alguna para eliminar los puntos negros de la ciudad
Tabla 2. Principales lugares (puntos negros) con mayor número de fallecidos.

\begin{tabular}{llc}
\hline Ubicación & \multicolumn{1}{c}{ Distrito } & $\begin{array}{c}\text { N. }{ }^{\circ} \text { de } \\
\text { fallecidos }\end{array}$ \\
\hline $\begin{array}{l}\text { Av. Panamericana Norte } \\
-1 .^{\circ} \text { de Pro }\end{array}$ & Comas & 5 \\
$\begin{array}{l}\text { Av. } .^{\circ} \text { de Mayo }- \\
\text { Mercado Perales }\end{array}$ & Santa Anita & 4 \\
$\begin{array}{l}\text { Carretera Central } \\
\text { km 13,5 }\end{array}$ & Ate Vitarte & 4 \\
$\begin{array}{l}\text { Av. Alfredo Mendiola }- \\
\text { Santa Rosa }\end{array}$ & Independencia & 3 \\
$\begin{array}{l}\text { Puente Nuevo } \\
\text { auxiliar bajo }\end{array}$ & El Agustino & 3 \\
\hline
\end{tabular}

Fuente: Secretaría Técnica del Consejo de Transporte de Lima y Callao

pese a que desde hace varios años atrás la Secretaría Técnica del Consejo de Transporte de Lima y Callao ha detectado los puntos negros de la ciudad de Lima, es decir, las zonas donde recurren los accidentes de tránsito, así como las zonas donde suceden accidentes con saldo fatal; las principales zonas se ubican en las zonas periféricas de la ciudad, tal como se observa en la Tabla $2^{(9)}$.

Existen otros puntos negros ubicados en distintos distritos de la ciudad de Lima como Santiago de Surco o San Martín de Porres, pero pese a que de acuerdo con la Ley N. ${ }^{\circ} 27972$, Ley Orgánica de Municipalidades, corresponde a las municipalidades normar, regular, organizar y mantener los sistemas de señalización y semáforos además de regular el tránsito urbano de peatones y vehículos, la Municipalidad Metropolitana de Lima (parte del Consejo Nacional de Seguridad Vial) no ha mostrado preocupación en desarrollar mecanismos que reduzcan la siniestralidad en dichas zonas de la ciudad. Esto es más grave pues en su oportunidad el Consejo de Transporte de Lima y Callao remitió este estudio a dicha Municipalidad y la Defensoría del Pueblo recomendó a la misma corporación municipal estudiar y eliminar los puntos negros, estos, a la fecha, no han sido eliminados.

Por ello, es imperativo un mayor compromiso de los gobiernos regionales y locales con la seguridad vial, desarrollando las líneas de trabajo establecidas en el Plan Nacional de Seguridad Vial.

\section{A MODO DE CONCLUSIÓN}

La Defensoria del Pueblo en su trabajo vinculado con la seguridad vial ha detectado varios problemas, uno de ellos es la falta de una adecuada estadística de 
accidentes de tránsito que permitan al Estado adoptar medidas que conlleven a la reducción de los accidentes de tránsito en el país.

En adición, se advierte la inacción del Consejo Nacional de Seguridad Vial y la falta de integración de los diferentes niveles del Estado en el desarrollo de las líneas de trabajo establecidas en el Plan Nacional de Seguridad Vial 2007-2011, que permitan un trabajo articulado de la seguridad vial en los estamentos del Estado.

\section{Conflictos de Interés}

El autor declara no tener conflictos de interés en la publicación de este artículo.

\section{REFERENCIAS BIBLIOGRÁFICAS}

1. Organización Mundial de la Salud. Informe mundial sobre prevención de los traumatismos causados por el tránsito. Ginebra: OMS; 2004.

2. Nantulya VM, Reich MR. The neglected epidemic: road traffic injuries in developing countries. BMJ. 2002; 324(7346): 1139-41.
3. Defensoría del Pueblo. Informe Defensorial № 137: El transporte urbano en Lima Metropolitana: Un desafío en defensa de la vida. Lima: Defensoría del Pueblo; 2008.

4. Consejo de Transporte de Lima y Callao. La vulnerabilidad de los peatones en la vialidad del área Metropolitana de Lima y Callao. Lima: Ministerio de Transportes y Comunicaciones; 2009.

5. Defensoría del Pueblo. Informe Defensorial $N^{\circ} 108$. Pasajeros en riesgo: la seguridad en el transporte interprovincial. Lima: Defensoría del Pueblo; 2008.

6. Perú, Ministerio de Transportes y Comunicaciones. Consejo Nacional de Seguridad Vial. Lima: MTC; 2008.

7. Chile, Comisión Nacional de Seguridad de Tránsito. Cuenta anual 2008. Comisión Nacional de Seguridad de Tránsito. Santiago: Ministerio de Transportes y Telecomunicaciones; 2009.

8. Perú, Consejos Nacional de Seguridad Vial. Plan nacional de seguridad vial 2007-2011. Lima: MTC; 2007.

9. Secretaria Técnica del Consejo de Transporte de Lima y Callao. Análisis de accidentes de tránsito en el área central de Lima y Callao - Información base del año 2006. Lima: MTC; 2008.

Correspondencia: Freddy Sagástegui Cruz

Correo electrónico: fsagastegui@defensoria.gob.pe

\section{Consulte las ediciones anteriores de la Revista Peruana de Medicina Experimental y Salud Pública en www.scielo.org.pe}

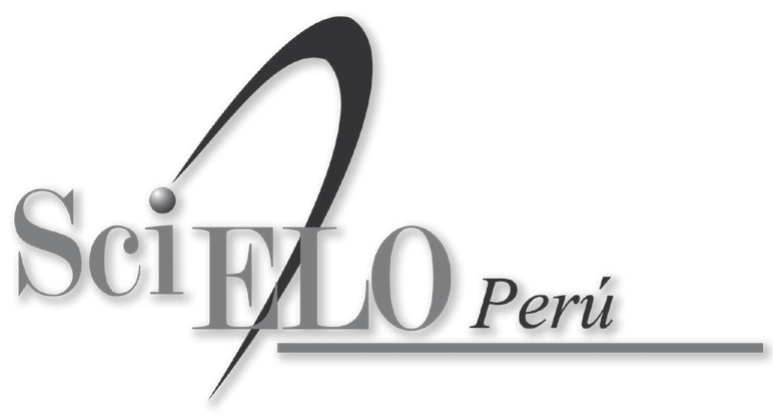

